\title{
Novel pantothenate derivatives for anti-malarial chemotherapy
}

\author{
Helmi E Pett ${ }^{1}$, Patrick AM Jansen ${ }^{2}$, Pedro HH Hermkens ${ }^{6}$, Peter NM Botman ${ }^{4}$, Christien A Beuckens-Schortinghuis ${ }^{4}$, \\ Richard H Blaauw ${ }^{4}$, Wouter Graumans ${ }^{1}$, Marga van de Vegte-Bolmer ${ }^{1}$, Karin MJ Koolen ${ }^{5}$, Floris PJT Rutjes ${ }^{3,6}$, \\ Koen J Dechering ${ }^{5}$, Robert W Sauerwein ${ }^{1,5}$ and Joost Schalkwijk $k^{2,6^{*}}$
}

\begin{abstract}
Background: A number of synthetic pantothenate derivatives, such as pantothenamides, are known to inhibit the growth of the human malaria parasite Plasmodium falciparum, by interfering with the parasite Coenzyme A (CoA) biosynthetic pathway. The clinical use of pantothenamides is limited by their sensitivity to breakdown by ubiquitous human pantetheinases of the vanin family.

Methods: A number of pantothenate derivatives (pantothenones) with potent and specific inhibitory activity against mammalian vanins were tested in a proliferation assay of asexual P. falciparum blood stages alone, and in combination with pantothenamides.

Results: The vanin inhibitors were found to protect pantothenamides against breakdown by plasma vanins, thereby preserving the in vitro anti-malarial activity. Moreover, some of the vanin inhibitors showed in vitro anti-malarial activity in the low micromolar range. The most potent antimalarial in this series of compounds (RR8), was found to compete with pantothenate in a combination proliferation assay. No correlation, however, was found between anti-vanin and anti-malarial activity, nor was pantetheinase activity detected in $P$. falciparum extracts.

Conclusions: Growth inhibition is most likely due to competition with pantothenate, rather than pantetheinase inhibition. As vanin inhibitors of the pantothenone class are stable in biological fluids and are non-toxic to mammalian cells, they may represent novel pantothenate-based anti-malarials, either on their own or in combination with pantothenamides.
\end{abstract}

Keywords: Malaria, Anti-malarial, Plasmodium falciparum, Pantothenate, Pantothenic acid, Pantothenamide, Pantothenone, Coenzyme A

\section{Background}

Each year over half a million people die of malaria, with Plasmodium falciparum being the primary cause of fatal malaria cases [1]. As the eradication of malaria is threatened by occurrence of clinical resistance to artemisinin derivatives, new drugs for malaria are sorely needed and so the search for new lead compounds continues [1].

Based on the observation, that addition of calcium pantothenate to Plasmodium lophurae cultures increased parasite viability, a selection of analogues of pantothenate

\footnotetext{
* Correspondence: joost.schalkwijk@radboudumc.nl

${ }^{2}$ Department of Dermatology and Radboud Institute for Molecular Life Sciences, Radboud University Nijmegen Medical Center, Nijmegen, The Netherlands

${ }^{6}$ Pansynt B V, Nijmegen, The Netherlands

Full list of author information is available at the end of the article
}

(pantothenic acid, vitamin B5), were tested for antiplasmodial activity as early as the 1940s [2]. These compounds included pantoyltaurine, substituted pantoyltaurylamides, sulphonamides, and pantothenones, according to the nomenclature used in a review on this subject by Spry et al. [3]. These and similar compounds were tested in different in vitro and in vivo malaria models from the 1960s and 1970s [4,5]. In 1976, Trager and Jensen published an article describing the continuous culture of P. falciparum [6], allowing Divo et al. to discover that pantothenate is indeed the only water soluble vitamin that needs to be exogenously available for $P$. falciparum survival [7]. Meanwhile, Clifton et al. prepared a series of analogues with the general structure N1-(substituted) pantothenamide, and 
found them to have antibacterial activity due to being antimetabolites of pantothenate [8].

Recent studies showed that some of the pantothenamides were also active against $P$. falciparum in vitro, provided that plasma pantetheinase activity was reduced [9]. This was discovered due to the observation that 'aging' $P$. falciparum growth media increased the anti-malarial activity of some pantothenamides [9]. Later, this same effect was achieved with heat inactivation of the parasite growth medium by de Villiers et al. [10]. The mechanism of breakdown of pantothenamides by pantetheinases of the vanin family was elucidated in detail by Jansen et al. who discovered that combining pantothenamides with small molecule vanin inhibitors, protected pantothenamides against breakdown, thereby dramatically increasing their antibacterial activity against both Staphylococcus aureus and Escherichia coli [11-14]. It has also been shown by de Villiers et al. that small modifications of the pantothenamide core structure could protect the molecule against pantetheinase-mediated degradation, albeit at a cost of a 100-fold decrease in antimalarial potency [10].

Compounds, such as the pantothenamides in E. coli or the fungal product CJ-15,801 in S. aureus may hijack Coenzyme A (CoA) biosynthesis, being phosphorylated in the first step of the biosynthesis by pantothenate kinase (PanK) and eventually blocking CoA production or interfering with fatty acid synthesis downstream along the pathway [15-17]. Almost a decade ago, the fungal product CJ-15,801, was also discovered to have modest anti-malarial activity against asexual intra-erythrocytic stages of $P$. falciparum in vitro, and was demonstrated to inhibit parasite growth by a mechanism related to CoA biosynthesis or utilization [18].

In this study a selection of novel pantetheine analogues of the pantothenone class were investigated for potential use as anti-malarial chemotherapy. The investigated compounds are shown to be conceptually promising either as a monotherapy or in a combination of drugs.

\section{Methods}

\section{Compounds}

Pantothenol was purchased from Sigma-Aldrich. Detailed synthesis procedures of CJ-15,801 and CXP14.1060 are provided as Additional file 1. The methodologies for synthesis of CXP14.1-034, RR2, RR6, RR7, RR8 and SN12,601 have been previously published in Jansen et al. [13]. N5-Pan and N7-Pan were synthesized as described in the supplemental material of Jansen et al. [14]. N9Pan was synthesized as N5-Pan and N7-Pan, but instead of using a pentylamine or heptylamine, a nonylamine was used for the synthesis of N9-Pan. The synthesis of SN14,621 and SN14,622 was performed as described in Winterbottom et al. [19]. The synthesis of phenethylPan was performed as described by Spry et al. [9].
Chemical structures for all of the compounds in this study are presented in Figure 1.

\section{Plasmodium falciparum culture}

The asexual stages of the NF54 strain P. falciparum were cultured as previously described [6], utilizing a shaker system with automated media change twice a day, parasites were kept in continuous culture within adapted Erlenmeyer flasks [20]. Erythrocytes were refreshed every two or three days to adjust haematocrit to $5 \%$ and parasitaemia to $0.5 \%$. Human erythrocytes (blood type A) were obtained from healthy blood donors, with no history of malaria. Culture media consisted of RPMI 1640 with HEPES [5.94 g/l, hypoxanthine $[0.05 \mathrm{~g} / \mathrm{l}], 10 \%(\mathrm{v} / \mathrm{v})$ pooled human serum (blood type A) obtained similarly to erythrocytes, and $0,2 \%(\mathrm{w} / \mathrm{v})$ sodium bicarbonate. Temperature was set to $37^{\circ} \mathrm{C}$ and a low oxygen gas mixture was constantly flushed over the culture maintaining a stable atmosphere of $3 \% \mathrm{O}_{2}, 4 \% \mathrm{CO}_{2}$, and $93 \% \mathrm{~N}_{2}$.

\section{Vanin activity assay}

Vanin/pantetheinase activity assay with aminomethylcoumarine (AMC) substrate and fluorescence readout was performed as described previously in Jansen et al. $[13,21]$. Human serum was used as a source of vanin enzymatic activity in assays to determine the anti-vanin activity of study compounds CJ-15,801, SN 12,601, SN 14,621, SN 14,622, CXP14.1-034, CXP14.1-060, RR2, RR6, RR7, and RR8. To determine whether P. falciparum parasites harbour vanin activity, assays were performed on parasite extracts. To this end, 5 to $9 \times 10^{8}$ non-synchronous asexual NF54 strain P. falciparum parasites were pelleted by centrifugation at $4000 \mathrm{rpm}$ for 10 minutes. Pellets were re-suspended in $5 \mathrm{ml}$ of $0.06 \%$ saponin in phosphate buffered saline (PBS) and incubated on ice for 5 minutes to remove erythrocytes. After this they were washed with PBS twice, with centrifugation in between as when pelleting cultures. Pellets from four different cultures were individually resuspended in a total volume of $300 \mu \mathrm{l}$ of PBS and lysed by sonication (6 $\times 3$ seconds). Vanin activity was determined by combining $29 \mu$ of lysate with $1 \mu \mathrm{l}$ of AMC substrate (final concentration of $333 \mu \mathrm{M}$ ) and incubation at room temperature. At 0,1 and 19 hours, $3 \mu \mathrm{l}$ of the reaction was diluted with $997 \mu \mathrm{l}$ of PBS and fluorescence was measured in a $200 \mu \mathrm{l}$ aliquot. Assay negative controls consisted of $29 \mu \mathrm{l}$ of PBS and $1 \mu \mathrm{l}$ of AMC substrate. Positive control consisted of $19 \mu \mathrm{l}$ of PBS, $10 \mu \mathrm{l}$ of human serum, and $1 \mu \mathrm{l}$ of AMC substrate.

\section{Plasmodium falciparum asexual blood stages assay with SYBR Green read-out}

A non-synchronous asexual NF54 strain P. falciparum culture was adjusted to a parasitaemia of $0.5-1 \%$ and a 


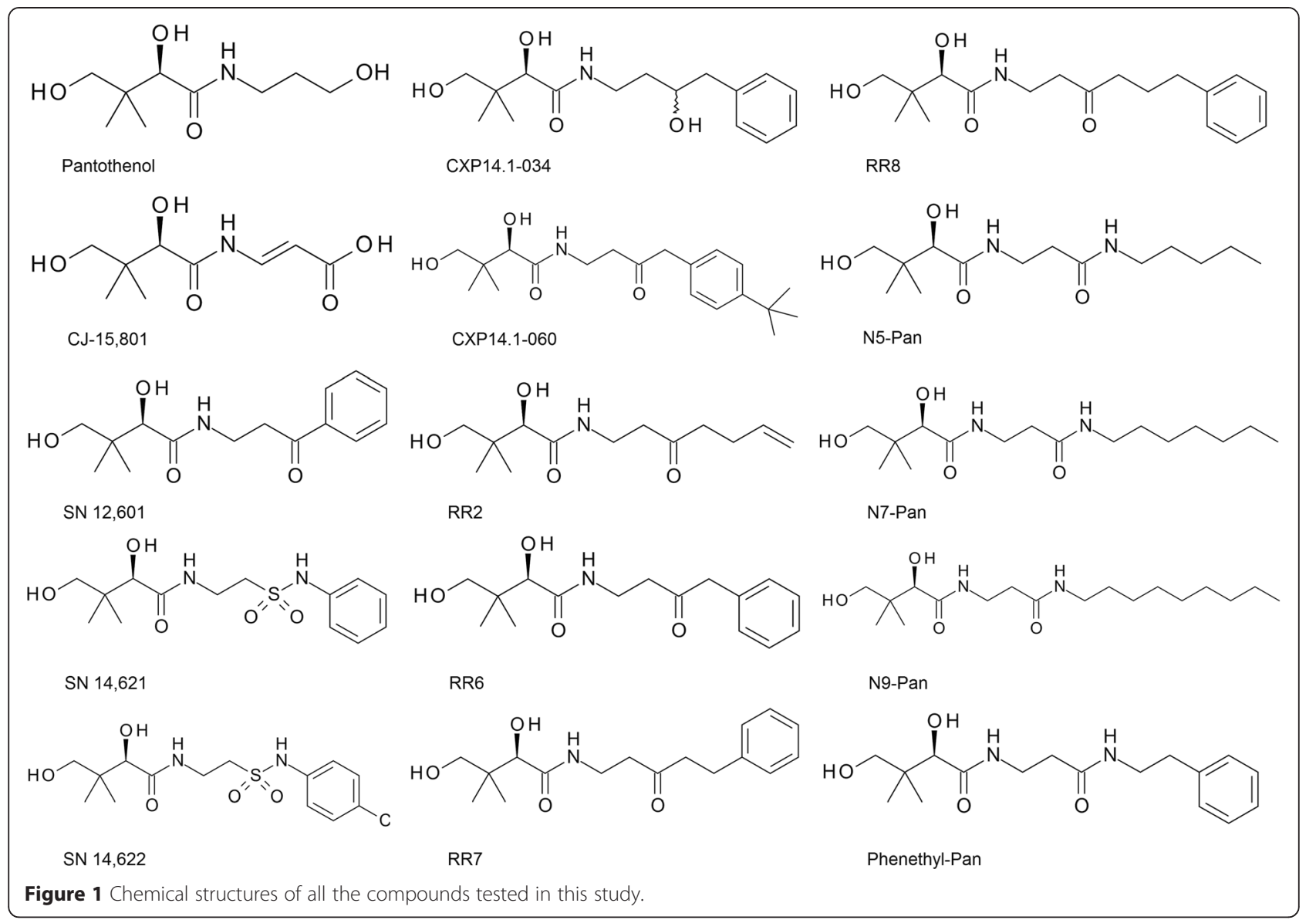

haematocrit of 1-5\%. The compound dilution curves were prepared in dimethylsulphoxide (DMSO) from $100 \mathrm{mM}$ to $10 \mu \mathrm{M}$ at half/log step dilutions. The established antimalarial dihydroartemisinin (DHA) was used as a positive control and diluted in DMSO from $1 \mathrm{mM}$ to $100 \mathrm{nM}$ at half/log step dilutions. The DMSO dilutions were 500-fold diluted in growth medium to yield a final DMSO concentration of $0.2 \%(\mathrm{v} / \mathrm{v})$, and final compound concentrations from $200 \mu \mathrm{M}$ to $20 \mathrm{nM}(2 \mu \mathrm{M}$ to $200 \mathrm{pM}$ for DHA). Higher concentrations for the experimental compounds would not have been possible to achieve due to limitations in final DMSO concentration $(0.1 \% \mathrm{v} / \mathrm{v})$. Fifty $\mu \mathrm{l}$ of each compound dilution was combined with $50 \mu \mathrm{l}$ of parasite culture in a black, clear-bottomed or entirely black 96-well plate. The outermost wells were filled with sterile water to prevent evaporation. DHA at a concentration of $1 \mu \mathrm{M}$ and $0.1 \%$ DMSO (negative control) were used to determine the assay window. The 96-well plates were incubated for 72 hours in a candle jar at $37^{\circ} \mathrm{C}$. Read-out was done using the DNA-marker SYBR Green as described previously [22,23]. All assays were conducted at least in triplicate.

When combining two compounds in the same well the conditions were otherwise similar to the single compound assays, however the final DMSO concentration was $0.2 \%$, which was also adjusted for the positive and negative controls and was not found to cause differences in results obtained with single compounds in assay containing $0.1 \%$ DMSO. The pantothenamide phenethyl-Pan was combined with a $10^{-7}$ to $10^{-5} \mathrm{M}$ concentration curve of CXP14.1-060; the other pantothenamides were combined only with a $5 \mu \mathrm{M}$ concentration of the same vanin inhibitor. The pantothenone vanin inhibitor RR8 was additionally combined with $20 \mu \mathrm{M}, 6.3 \mu \mathrm{M}, 2.0 \mu \mathrm{M}$, and $0.6 \mu \mathrm{M}$ of pantothenate to explore potential competition for the same molecular target in P. falciparum. All assays were conducted at least in triplicate.

\section{Statistical analyses}

Statistical analyses were performed using GraphPad Prism 5. This includes producing inhibition curves, normalizing them to percentage of inhibition, calculation of $\mathrm{IC}_{50}$ values, correlation coefficients (Spearman nonparametric correlation), and Schild analyses [24]. The $\mathrm{IC}_{50}$ is the concentration of the compound in question at which the compound reaches $50 \%$ inhibition relative to the DHA control curve, and is calculated using the interpolate function in GraphPad Prism 5. 


\section{Results}

Anti-malarial activity of pantothenamides

Using P. falciparum cultured in 10\% human serum, the low level of anti-malarial activity of the pantothenamides N5-Pan, N7-Pan and phenethyl-Pan was confirmed, as shown in Figure 2A. None of these pantothenamides showed an $\mathrm{IC}_{50}$ below $50 \mu \mathrm{M}$. In addition, N9-Pan was tested. N9-Pan is a pantothenamide that has previously been shown to have modest antibacterial activity but had not been investigated for its anti-malarial activity [8]. N9-Pan was found to be clearly more potent than the other pantothenamides, with an $\mathrm{IC}_{50}$ of about $15 \mu \mathrm{M}$, in the presence of serum (Figure 2A). To investigate the effect of vanin inhibition on the potency of the pantothenamides N5-Pan, N7-Pan and N9-Pan, these

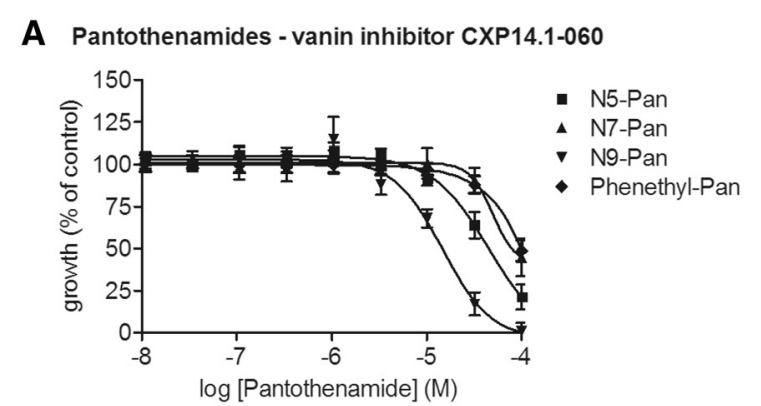

B Pantothenamides $+5 \mu \mathrm{M}$ vanin inhibitor CXP14.1-060

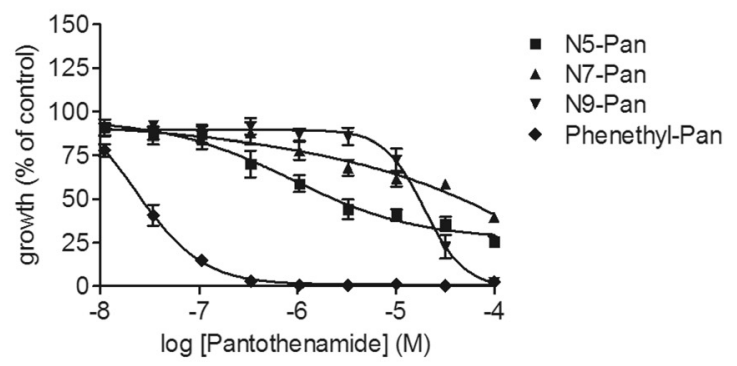

C DHA +/- $5 \mu \mathrm{M}$ vanin inhibitor CXP14.1-060

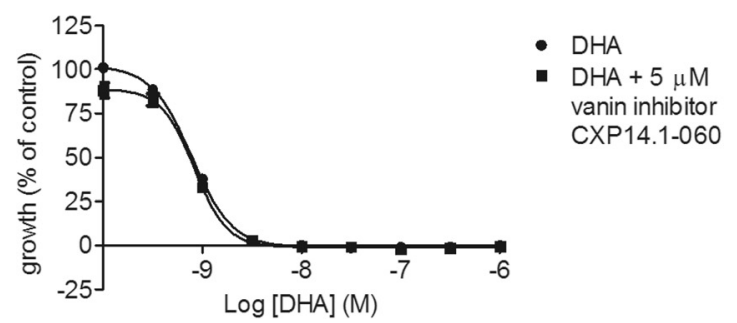

Figure 2 Anti-malarial activity of pantothenamides. Four pantothenamides, phenethyl-Pan, but also N5-Pan, N7-Pan and N9-Pan in asexual blood-stages proliferation assay with SYBR Green read-out, without and in combination with $5 \mu \mathrm{M}$ of vanin inhibitor CXP14.1-060. A) N5-Pan, N7-Pan, N9-Pan and phenethyl-Pan without vanin inhibitor CXP14.1-060. B) N5-Pan, N7-Pan, N9-Pan and phenethyl-Pan in combination with vanin inhibitor CXP14.1-060. C) DHA with and without $5 \mu \mathrm{M}$ of vanin inhibitor CXP14.1.-060. were combined with $5 \mu \mathrm{M}$ of the newly synthesized vanin inhibitor CXP14.1-060, that has nanomolar potency against human vanins, yet no anti-malarial activity up to the highest concentration tested $(100 \mu \mathrm{M})$ (Table 1$)$. The potency of N5-Pan and N7-Pan increased upon vanin inhibition, but the effect was not as great as with phenethyl-Pan (Figure 2B). Remarkably, the potency of N9-Pan was not increased by the addition of the vanin inhibitor (Figure $2 \mathrm{~B}$ ). The potency of DHA did not change upon addition of $5 \mu \mathrm{M}$ of the vanin inhibitor CXP14.1-060 (Figure 2C).

Phenethyl-Pan was selected to further investigate the protective effect of the synthetic vanin inhibitor CXP14.1-060 on its anti-malarial activity. Phenethyl-Pan was previously shown to have an $\mathrm{IC}_{50}$ of $20 \mathrm{nM}$ in 'aged' medium and is the most potent anti-malarial pantothenamide known so far [9]. A dose range of this compound was tested in the presence of varying concentrations of CXP14.1-060 (Figure 3). The potency of phenethyl-Pan visibly improved upon addition of increasing concentrations of CXP14.1-060, reaching an $\mathrm{IC}_{50}$ of $23 \mathrm{nM}$ (95\% CI: 16.5-31.9 $\mathrm{nM}$ ) upon addition of $10 \mu \mathrm{M}$ of CXP14.1-060 (Figure 3). In the presence of $10 \mu \mathrm{M}$ of vanin inhibitor CXP14.1-060, phenethyl-Pan showed a full inhibition of parasite growth, comparable to the efficacy of DHA, which was used as a reference compound and has an $\mathrm{IC}_{50}$ in the low nanomolar range. This experiment shows that small molecule inhibition of pantothenamide hydrolysis will allow the same level of protection found in heatinactivated medium as shown by the comparable $\mathrm{IC}_{50}$ values [10].

Table 1 IC $_{50}$ values for individual compounds

\begin{tabular}{|c|c|c|c|}
\hline \multirow[b]{2}{*}{ Compound } & \multicolumn{2}{|c|}{ Anti-malarial activity } & \multirow{2}{*}{$\begin{array}{l}\text { Anti-vanin activity* } \\
\mathrm{IC}_{50}(\mu \mathrm{M})\end{array}$} \\
\hline & $\mathrm{IC}_{50}(\mu \mathrm{M})$ & 95\% C.I. $(\mu \mathrm{M})$ & \\
\hline Pantothenol & $>100$ & NA & NT \\
\hline CJ-15,801 & $>100$ & NA & 213.60 \\
\hline SN 12,601 & 6.1 & $4.5-8.7$ & 17.61 \\
\hline SN 14,621 & 33.6 & 7.3-NA & 33.70 \\
\hline SN 14,622 & 7.4 & $3.5-20.7$ & 31.89 \\
\hline CXP14.1-034 & 24.1 & 11.3-NA & 1.69 \\
\hline CXP14.1-060 & $>100$ & NA & 0.039 \\
\hline RR2 & 2.6 & $2.1-3.3$ & 2.85 \\
\hline RR6 & 14.5 & 5.3-NA & 0.04 \\
\hline RR7 & 7.7 & $5.1-12.6$ & 0.11 \\
\hline RR8 & 2.2 & $1.6-3.1$ & 0.27 \\
\hline N5-Pan & 45.1 & $34.8-60.2$ & NT \\
\hline N7-Pan & 79.0 & 34.0-NA & NT \\
\hline N9-Pan & 14.6 & $11.0-20.1$ & NT \\
\hline Phenethyl-Pan & 98.2 & NA & NT \\
\hline
\end{tabular}

*Anti-vanin activity tested in human serum.

NA: Not Applicable.

NT: Not Tested. 


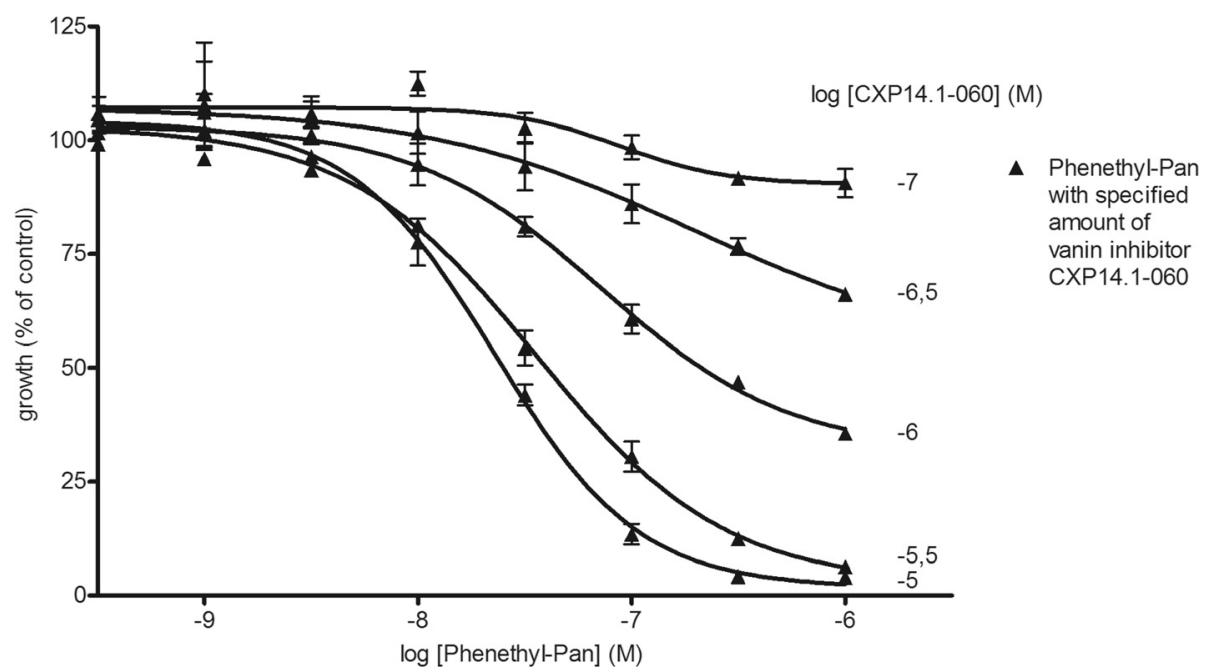

Figure 3 Anti-malarial activity of phenetyl-Pan in the presence of varying concentrations of vanin inhibitor CXP14.1-060. Combination of anti-malarial pantothenamide phenethyl-Pan [9] with novel vanin inhibitor CXP14.1-060. Filled upright triangles represent phenethyl-Pan, with CXP14.1-060 added at a different concentration for every curve as indicated in figure. At a concentration of $10 \mu \mathrm{M}$ of CXP14.1-060 phenetyl-Pan has an $\mathrm{IC}_{50}$ of $23 \mathrm{nM}(95 \% \mathrm{Cl}: 16.5-31.9 \mathrm{nM})$.

\section{Anti-malarial activity of pantothenones}

15 pantothenate derivatives were synthesized and assayed for anti-malarial activity in the in the presence of $10 \%$ human serum. The structures of these compounds are presented in Figure 1, and their observed individual biological effects are presented in Table 1 . Some of the newly synthesized vanin inhibitors were structurally similar to pantothenate derivatives described in the 1940s, which were shown to have anti-malarial activity in avian malaria models [2]. Some of these reference compounds were resynthesized, including the pantothenone SN12,601 and the sulphonamides SN 14,621 and SN 14,622. In this study, they were found to have moderate activity, and the effect of their dilution curves on $P$. falciparum growth are shown in Figure 4. In addition, pantothenol and CJ-15,801, two natural compounds with known weak activity against $P$. falciparum, were also tested $[18,25]$. CJ-15,801 was found to be a poor inhibitor of $P$. falciparum growth (Table 1 and Figure 4) and pantothenol showed no anti-malarial activity up to the highest concentration tested $(100 \mu \mathrm{M})$ (Table 1$)$. Out of the newly synthesized pantothenones, the vanin inhibitor RR8 was the most potent of these compounds, having an $\mathrm{IC}_{50}$ value of $2.2 \mu \mathrm{M}$ (95\% CI: 1.6-3.1 $\mu \mathrm{M}$ ) (Table 1 and Figure 4).

\section{Mechanism of anti-malarial activity of pantothenones}

It was considered, that the pantetheinase inhibiting activity of the new compounds may contribute to antimalarial activity. Therefore, the $\mathrm{IC}_{50}$ values of in vitro inhibition of asexual blood stages of P. falciparum were plotted against the $\mathrm{IC}_{50}$ values of anti-vanin activity in human serum (Figure 5). There was no correlation between these activities, suggesting that pantetheinase inhibition is not the mode of action of the small molecule inhibitors in P. falciparum asexual blood stages. In line with this finding, an assay using the AMC substrate to detect pantetheinase activity, did not detect hydrolytic activity in extracts of purified asexual stages of $P$. falciparum, even after a $19 \mathrm{~h}$ incubation period, suggesting that the parasite lacks such enzyme activity (Figure 6). This notion was corroborated by a BLAST search that did not reveal sequences in the $P$. falciparum genome database with homology to mammalian vanin genes. In order to investigate an alternate mode of action, competition experiments with pantothenate were performed. To this end, a P. falciparum asexual blood stage growth assay was performed with eight concentrations of RR8 combined with eight concentrations of pantothenate. An increase in the $\mathrm{IC}_{50}$ of $\mathrm{RR} 8$ was found to occur at addition of increasing concentrations of pantothenate (Figure 7A). Upon performing a Schild analysis on the results of the concentrations, $20 \mu \mathrm{M}, 6.3 \mu \mathrm{M}, 2.0 \mu \mathrm{M}$, and $0.6 \mu \mathrm{M}$ of pantothenate in combination with a dilution curve of RR8, the slope of the line in the Schild Plot was $1.087 \pm 0.089$, indicating that RR8 and pantothenate are competing for the same target in P. falciparum (Figure 7B).

\section{Discussion}

This study underscores the potential of pantothenate derivatives for anti-malarial therapy, and demonstrates that the most potent serum-labile anti-malarial pantothenamide (phenethyl-Pan) can be effectively protected against hydrolysis by serum pantetheinases using the novel vanin inhibitor CXP14.1-060. From a mechanistic point of view, this study indicates that the pantothenone RR8 


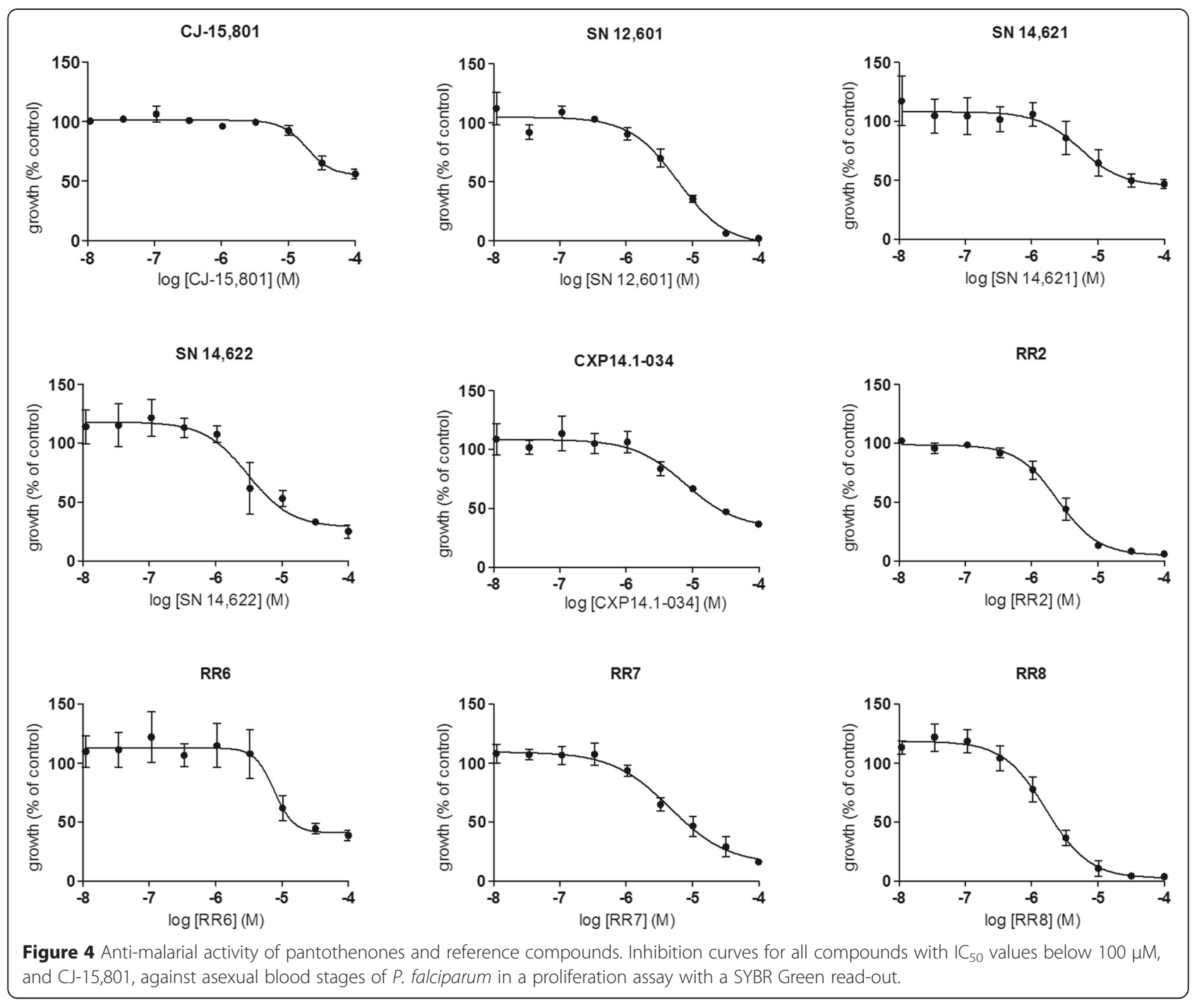

exerts its anti-malarial effect through competition with pantothenate.

In the 1940s, a number of chemical variations on pantothenate were synthesized and tested for anti-malarial activity [2]. These included pantothenones and sulphonamides, which were found to be active against avian malaria. This study shows that these compounds are also active against the human parasite P. falciparum.

Although the new vanin inhibitor CXP14.1-060 effectively protected phenethyl-Pan, such a combination of drugs would be undesirable from a drug development perspective. Clearly, the potency and/or stability of pantothenate derivatives needs to be improved before they can enter a drug development programme as therapeutic agents for human malaria infection. Nevertheless, the recent discovery of phenethyl-Pan with an $\mathrm{IC}_{50}$ of $20 \mathrm{nM}$ is encouraging [9]. Although this compound is unstable in plasma, it illustrates that it is realistic and feasible to aim for pantothenate derivatives active in the low nanomolar range. The study by de Villiers et al. showed that structural modifications of pantothenamides can be introduced to confer resistance to plasma-mediated breakdown [10]. These novel compounds, although less potent than the original pantothenamides, are a starting point for further lead optimization studies [10]. Optimization of the potency would be important to maximize the risk-benefit of a novel drug, as side effects may be mediated by low-affinity, off-target effects. In addition, increasing the potency may lead to a lower effective dose in humans, and hence impact the cost of treatment. Availability of affordable medicines is an important driver for success in malaria control, and the goal for development of novel drug therapies is to achieve effective treatment with a total cost of US\$1 [26]. In that respect, the molecules described here provide attractive candidates as their chemistry is simple, which ensures a low cost of goods in a manufacturing process.

Many of the marketed anti-malarials and compounds in the clinical development portfolio originate from whole 


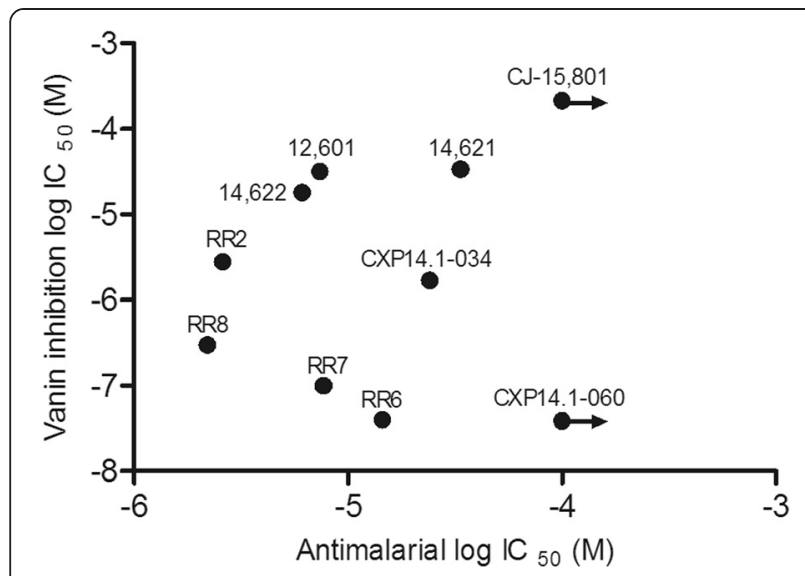

Figure $\mathbf{5}$ Lack of correlation between anti-malarial activity and anti-vanin activity. The $I_{50}$ for inhibition of vanin activity in human serum was plotted against the anti-malarial $I C_{50}$ in asexual blood stages proliferation assay of $P$. falciparum with SYBR Green read-out. No significant correlation was observed. (Spearman $r h o=0.06079(p=0.8651)$ ).

cell phenotypic screening efforts and exert their actions by inhibiting multiple targets or pathways of the parasite. Although such a polypharmacological profile may be important to their efficacy, it is an undesirable feature in a rational medicinal chemistry approach. The exact target of the anti-malarial pantothenate derivatives has not been identified unequivocally but is it likely that they exert their effects by affecting targets dependent on pantothenate. In theory, the observed effects could still be mediated by effects on red blood cell biology (e.g., red blood cell pantothenate kinases (PANK)) rather than directly on the parasite. However, the recent discovery of a parasitespecific pantothenate transporter [27] leaves very little

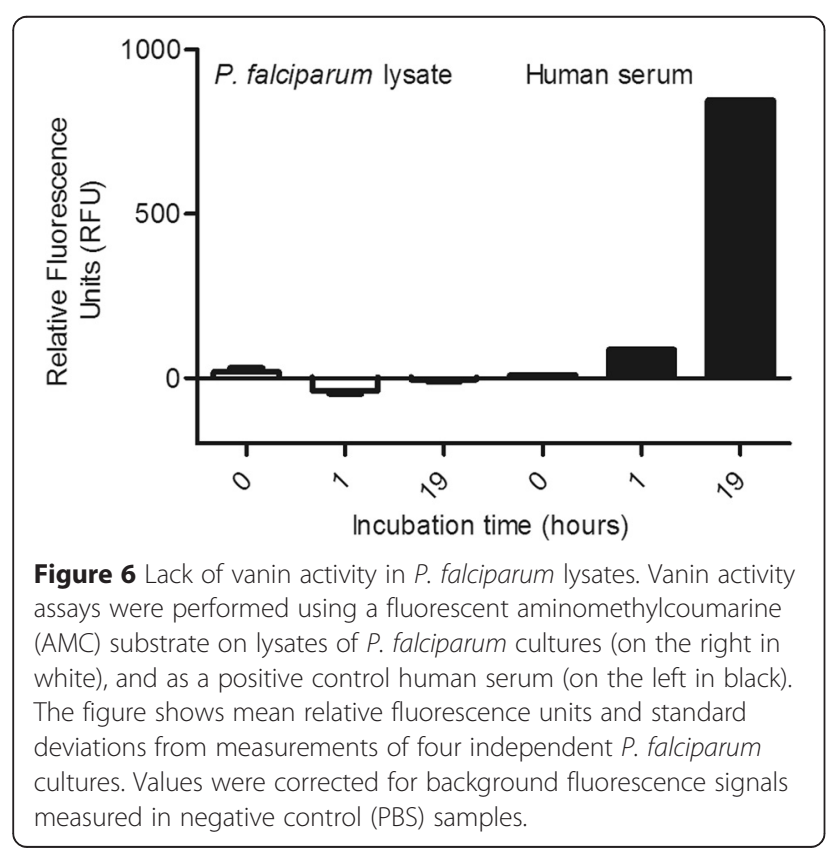

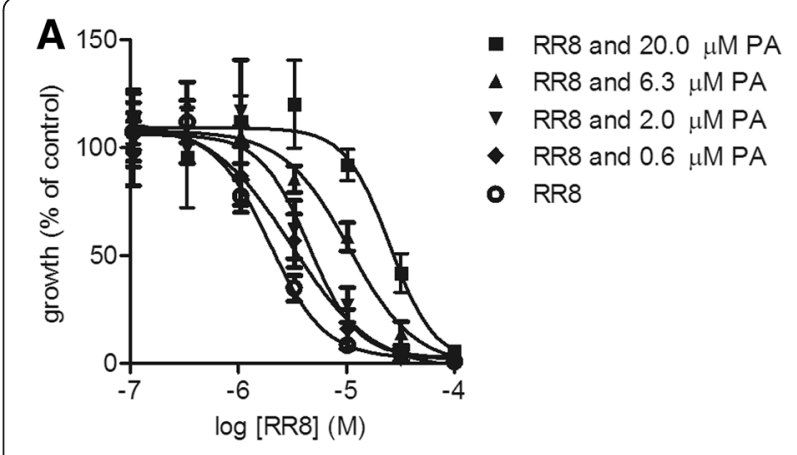

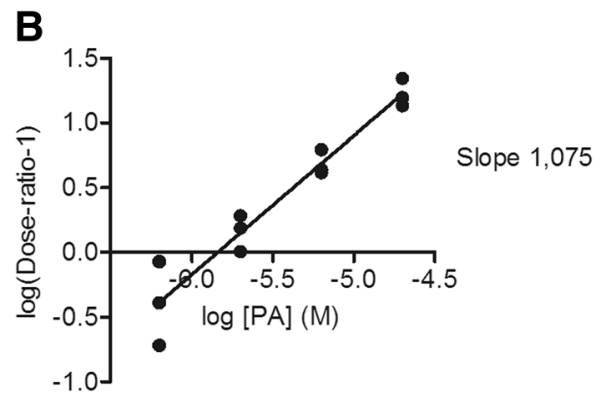

Figure 7 Pantothenate competes with RR8. Competition assay between RR8 and pantothenate (PA), results of Schild analysis. A) Shifting $I \mathrm{C}_{50}$ : Combined data points from three experiments. RR8 with $20.0 \mu \mathrm{M}$ PA, RR8 with $6.3 \mu \mathrm{M}$ PA, RR8 with $2.0 \mu \mathrm{M}$ PA, RR8 with $0.6 \mu \mathrm{M}$ PA and RR8 alone. B) Schild plot: Combined data points from three experiments. A slope of 1 is indicative of a competitive antagonistic relationship between RR8 and PA.

doubt that the parasite itself is the target of interfering with pantothenate dependent pathways. Future drug development efforts would benefit from information on the molecular targets of pantothenamides, which would include both biosynthetic pathways (CoA synthesis, lipid synthesis, energy metabolism) and pantothenate transport systems. Structural information, which is available for mammalian and bacterial PANK, could guide medicinal chemistry strategies to achieve specific inhibition of the parasite enzyme and reduce side effects on the host.

\section{Conclusions}

Pantothenamides with anti-malarial activity can be protected from breakdown by ubiquitous pantetheinases of the vanin family with small molecule pantothenone vanin inhibitors. Some of these pantothenones exhibit antimalarial activity in their own right. Compound series such as the one tested in this publication should be studied further for use as lead compounds for anti-malarial treatment.

\section{Additional file}

Additional file 1: Supplemental Materials and Methods. The supplemental materials and methods explain how the compounds CJ-15,801 and CXP14.1-060 were synthesized. 


\section{Abbreviations}

CoA: Coenzyme A; DHA: Dihydroartemisinin; DMSO: Dimethylsulphoxide; PA: Pantothenic acid, pantothenate, vitamin B5; PanK: Pantothenate kinase (Plasmodium falciparum); PANK: Pantothenate kinase (Homo sapiens).

\section{Competing interests}

KD and RS hold shares in TroplQ Health Sciences, a spin-off company of the RadboudUMC that aims to develop anti-malarial drugs. JS, FPJTR and PHHH hold shares in Pansynt, a spin-off company of the RadboudUMC that aims to develop pantothenate-based drugs for infectious diseases. Some of the compounds described in the manuscript are covered by a patent application (PCT/NL2011/050385) filed by the RadboudUMC (inventors: PAMJ, JS, RS, PHHH, FPJTR).

\section{Authors' contributions}

HEP carried out the majority of $P$. falciparum proliferation assays and the majority of the writing of the manuscript. PAMJ carried out the vanin inhibition assays and wrote some of the manuscript. $\mathrm{PHHH}$ is responsible for design of active compounds and for writing of the supplemental material of the manuscript. PNMB and CABS synthesized active compounds. RHB was responsible for conception of chemical synthesis routes. WG and MV-B worked on early experimentation with compounds. KMJK performed the experiment that resulted in Figure 2 C. FPJTR is responsible for design of active compounds. KD supervised experimental work of HEP and wrote a substantial part of the discussion section. RS approved work being done during experimental process. JS initiated the project, supervised experimental work of HEP and PAMJ, and wrote a substantial part of this publication. All authors have read and approved final version of the manuscript.

\section{Acknowledgements}

This work was supported by the Radboud University Nijmegen Medical Center and by a grant of The Netherlands Genomics Initiative, Grant no. 93611013. Also, we extend our gratitude to Martijn Timmerman for expert technical advice in design and construction of drug assays in 96-well plates.

\section{Author details}

'Department of Medical Microbiology, Radboud University Nijmegen Medical Center, Nijmegen, The Netherlands. ${ }^{2}$ Department of Dermatology and Radboud Institute for Molecular Life Sciences, Radboud University Nijmegen Medical Center, Nijmegen, The Netherlands. ${ }^{3}$ Radboud University Nijmegen, Institute for Molecules and Materials, Nijmegen, The Netherlands. ${ }^{4}$ Chiralix B V, Nijmegen, The Netherlands. ${ }^{5}$ TroplQ Health Sciences, Nijmegen, The Netherlands. ${ }^{6}$ Pansynt B V, Nijmegen, The Netherlands.

Received: 1 December 2014 Accepted: 4 April 2015

Published online: 18 April 2015

\section{References}

1. WHO. World malaria report 2013. Geneva: World Health Organization; 2013.

2. Wiselogle FY. A survey of antimalarial drugs. Ann Arbor, Michigan: Edwards, J.W.; 1946.

3. Spry C, Kirk K, Saliba KJ. Coenzyme a biosynthesis: an antimicrobial drug target. FEMS Microbiol Rev. 2008;32:56-106.

4. Trager W. Coenzyme a and the antimalarial action in vitro of antipantothenate against plasmodium lophurae, $P$. Coatneyi and $P$. Falciparum. Trans N Y Acad Sci. 1966;28:1094-108.

5. Trager W. Further studies on the effects of antipantothenates on malaria parasites (Plasmodium coatneyi and P. falciparum) in vitro. J Protozool. 1971:18:232-9.

6. Trager W, Jensen JB. Human malaria parasites in continuous culture. Science. 1976;193:673-5.

7. Divo AA, Geary TG, Davis NL, Jensen JB. Nutritional requirements of Plasmodium falciparum in culture. I. Exogenously supplied dialyzable components necessary for continuous growth. J Protozool. 1985;32:59-64.

8. Clifton G, Bryant SR, Skinner CG. N'-(substituted) pantothenamides, antimetabolites of pantothenic acid. Arch Biochem Biophys. 1970;137:523-8.

9. Spry C, Macuamule C, Lin Z, Virga KG, Lee RE, Strauss E, et al. Pantothenamides are potent, on-target inhibitors of Plasmodium falciparum growth when serum pantetheinase is inactivated. PLoS One. 2013;8, e54974.
10. de Villiers M. Structural modification of pantothenamides counteracts degradation by pantetheinase and improves antiplasmodial activity. ACS Med Chem Lett. 2013;4:784-9.

11. Jansen PA, Zeeuwen PL, Schalkwijk J, Rutjes FP, Ritzen B, Hermkens PH. Pantothenic acid derivatives and their use in the treatment of microbial infections. Patent Application number EP11725211, Publication number WO2011152720. 2011.

12. Jansen PA, Schalkwijk J, Rutjes FP, Sauerwein R, Hermkens PH. Derivatives of pantothenic acid and their use for the treatment of malaria. Patent application number EP11725211, publication number WO2011152721. 2011.

13. Jansen PA, van Diepen JA, Ritzen B, Zeeuwen PL, Cacciatore I, Cornacchia C, et al. Discovery of small molecule vanin inhibitors: new tools to study metabolism and disease. ACS Chem Biol. 2013:8:530-4.

14. Jansen PA, Hermkens PH, Zeeuwen PL, Botman PN, Blaauw RH, Burghout P, et al. Combination of pantothenamides with vanin inhibitors as a novel antibiotic strategy against Gram-positive bacteria. Antimicrob Agents Chemother. 2013:57:4794-800.

15. Strauss E, Begley TP. The antibiotic activity of N-pentylpantothenamide results from its conversion to ethyldethia-coenzyme a, a coenzyme a antimetabolite. J Biol Chem. 2002;277:48205-9.

16. Zhang YM, Frank MW, Virga KG, Lee RE, Rock CO, Jackowski S. Acyl carrier protein is a cellular target for the antibacterial action of the pantothenamide class of pantothenate antimetabolites. J Biol Chem. 2004:279:50969-75.

17. van der Westhuyzen R, Hammons JC, Meier JL, Dahesh S, Moolman WJ, Pelly SC, et al. The antibiotic CJ-15,801 is an antimetabolite that hijacks and then inhibits CoA biosynthesis. Chem Biol. 2012;19:559-71.

18. Saliba KJ, Kirk K. CJ-15,801, a fungal natural product, inhibits the intraerythrocytic stage of Plasmodium falciparum in vitro via an effect on pantothenic acid utilisation. Mol Biochem Parasitol. 2005;141:129-31.

19. Winterbottom R, Clapp JW, Miller WH, English JP, Roblin Jr RO. Studies in chemotherapy; amides of pantoyltaurine. J Am Chem Soc. 1947;69:1393-401.

20. Ponnudurai $\mathrm{T}$, Lensen AH, Meis JF, Meuwissen JH. Synchronization of Plasmodium falciparum gametocytes using an automated suspension culture system. Parasitology. 1986;93(Pt 2):263-74.

21. Ruan BH, Cole DC, Wu P, Quazi A, Page K, Wright JF, et al. A fluorescent assay suitable for inhibitor screening and vanin tissue quantification. Anal Biochem. 2010;399:284-92.

22. Smilkstein M, Sriwilaijaroen N, Kelly JX, Wilairat $P$, Riscoe M. Simple and inexpensive fluorescence-based technique for high-throughput antimalarial drug screening. Antimicrob Agents Chemother. 2004;48:1803-6.

23. Bennett TN, Paguio M, Gligorijevic B, Seudieu C, Kosar AD, Davidson E, et al. Novel, rapid, and inexpensive cell-based quantification of antimalarial drug efficacy. Antimicrob Agents Chemother. 2004;48:1807-10.

24. Wyllie DJ, Chen PE. Taking the time to study competitive antagonism. Br J Pharmacol. 2007;150:541-51.

25. Saliba KJ, Ferru I, Kirk K. Provitamin B5 (pantothenol) inhibits growth of the intraerythrocytic malaria parasite. Antimicrob Agents Chemother. 2005;49:632-7.

26. Burrows JN, van Huijsduijnen RH, Mohrle JJ, Oeuvray C, Wells TN. Designing the next generation of medicines for malaria control and eradication. Malar J. 2013;12:187.

27. Augagneur Y, Jaubert L, Schiavoni M, Pachikara N, Garg A, Usmani-Brown S, et al. Identification and functional analysis of the primary pantothenate transporter, PfPAT, of the human malaria parasite Plasmodium falciparum. J Biol Chem. 2013;288:20558-67.

\section{Submit your next manuscript to BioMed Central and take full advantage of:}

- Convenient online submission

- Thorough peer review

- No space constraints or color figure charges

- Immediate publication on acceptance

- Inclusion in PubMed, CAS, Scopus and Google Scholar

- Research which is freely available for redistribution 